\title{
Data acquisition and online control system for new gas-electron multiplier detectors in the endcap muon system of the CMS experiment
}

\author{
José David Ruiz-Álvarez* \\ On behalf of the CMS Muon Group \\ Universidad de los Andes ( $\mathrm{CO}$ ) \\ E-mail: jose.ruiz@cern.ch
}

\begin{abstract}
A new data acquisition and on-line control system is being developed for gas-electron multiplier (GEM) detectors which will be installed in the forward region $(1.6<|\eta|<2.2)$ of the CMS muon spectrometer during the 2nd long shutdown of the LHC, planned for the period 20182019. A prototype system employs the TOTEM VFAT2 ASIC that will eventually be replaced with the VFAT3 ASIC, under development. The front-end ASIC communicates over printed circuit lines with an intermediate on-detector board called the opto-hybrid. Data, trigger, and control information is transmitted via optical fiber between the opto-hybrid and an off-detector readout system using micro-TCA technology. On-line software, implemented in the CMS XDAQ framework, includes applications for latency and HV scans, and system management. We report on the operational status of the prototype system that has been tested using cosmic ray muons and extracted high-energy particle beams. This work is preparatory for the operation of a prototype GEM detector system to be installed in the CMS experiment in 2017.
\end{abstract}

38th International Conference on High Energy Physics

3-10 August 2016

Chicago, USA

\footnotetext{
* Speaker.
} 


\section{Introduction}

The CMS experiment has been preparing for the upgrade on 2021, the last LHC run before the High Luminosity LHC (HL-LHC). Part of the muon system upgrade will consist of the installation of a new Gas Electron Multiplier (GEM) detector in the endcap region, between $1.6<|\eta|<2.2$. This new detector is called GE1/1 corresponding to the first GEM endcap station located in the first muon station. This station will add redundancy to the muon measurement increasing the CMS trigger capabilities regarding muons. A detailed presentation of GE1/1 project can be found in [1].

A triple GEM technology was chosen for GE1/1 project. Details about the operational principle of this technology can be found in [2]. Such technology assures the rate performance needed for HL-LHC.

\subsection{GEM detectors at CMS}

The GE1/1 consists of two disks of GEM detectors one in each endcap. Each disk is formed of 36 superchambers, where each superchamber is constituted of two GEM chambers. Therefore, each superchamber covers around 10 degrees in the azimuthal angle.

\subsection{Electronics}

Each GE1/1 chamber has an approximate active area of $0.5 \mathrm{~m}^{2}$. This active area is covered with readout strips located in the GEM Electronic Board or GEB. The readout strips are taken out in 24 readout sectors. The data of each sector is collected by a VFAT2 font-end chips. An upgraded version of this chip is currently under development (VFAT3).

Each VFAT2 builds a data packet that is sent through an e-link to an on-detector component called opto-hybrid $(\mathrm{OH})$. The $\mathrm{OH}$ serializes the data and sends them to the off detector components via an optical link. The $\mathrm{OH}$ also receives the triggering information sent by the off-detector components of the system.

The off-detector electronics are the interface with CMS central systems: DAQ, trigger, etc. They are based on a $\mu$ TCA crate technology, that is the standard used for CMS upgrade. The $\mathrm{OH}$ sends the data to an AMC (Advanced Mezzanine Card) type card CTP7. The CTP7 sends the data to an AMC13 card through the $\mu$ TCA back-plane. The AMC13 card communicates directly with central CMS DAQ system.

\section{Data acquisition and online control system}

A DAQ system have been developed for the GE1/1 project. The basic tool for DAQ at CMS is the XDAQ platform [3]. Also various tools for data visualization, quality monitoring among other have been under development.

\subsection{DAQ development}

Figure 1 shows a diagram of the structure of all software components for GE1/1 DAQ system.

The infrastructure design basis are the managers. There is a manager that controls the CTP7, AMC13 and $\mathrm{OH}$ components. A global GEM supervisor is the interface between the user and the managers. From the GEM supervisor the user can control all the different electronic components of 


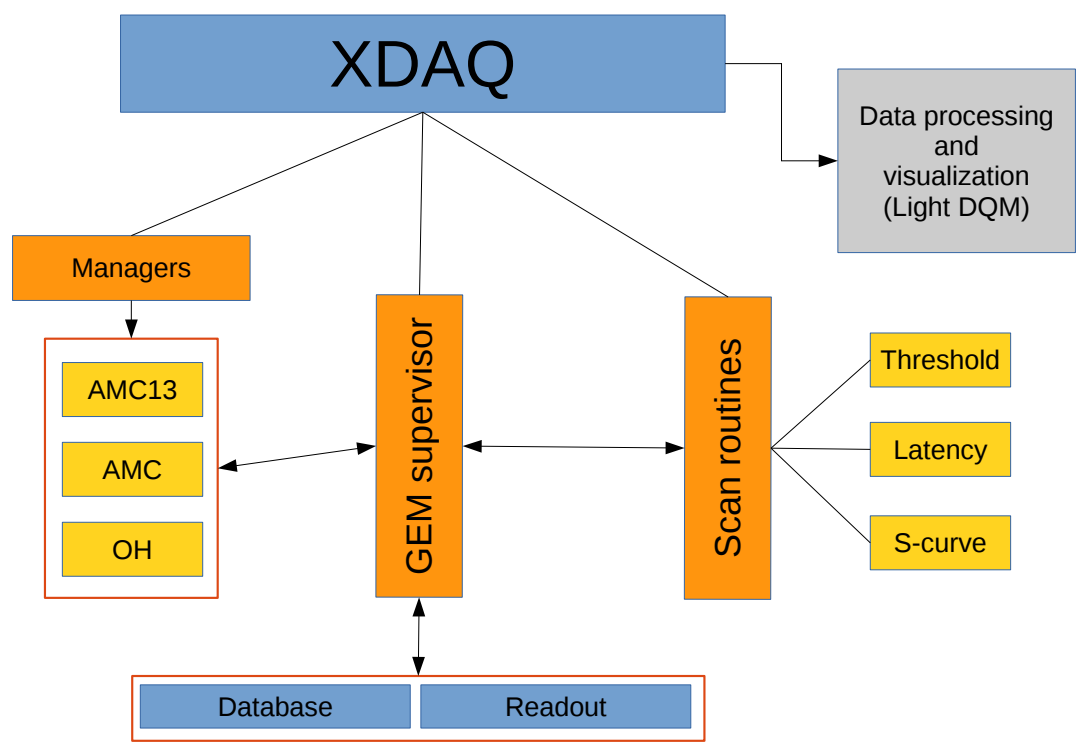

Figure 1: GE1/1 DAQ development structure.

the GE1/1 system for standard data taking or other purposes as scan routines. The GEM supervisor is also the interface to the GE1/1 database and readout.

Three scan routines have been developed: Threshold, Latency and S-curve. They can be launched from the GEM supervisor and is through it that the scan routines access the managers and write data using the system readout and database. Threshold and latency scans are designed to be done from the AMC13 and are performed on each VFAT2. A high voltage scan is also under preparation.

Each component manager controls the status of the corresponding electronic component. The $\mathrm{OH}$ manager also sets the parameters on the connected VFAT2 chips. The AMC13 manager controls also the trigger mode and TTC (Trigger, Timing and Control) source and also enables or disables the connected AMC cards.

The XDAQ platform allows the system to be controlled from an HTML application. Therefore, the user can control all the different components (managers, GEM supervisor, scans) via this HTML application.

Further development is being handled to communicate with central CMS systems. This development focus in the integration of the GE1/1 system in the RCMS (Run Control and Monitoring System).

\subsection{Data processing and visualization}

A database has been developed for GE1/1 system. It takes care of keeping all the information of settings applied to the system when running. Therefore, assuring a traceability of detector conditions during data taking. 
Various tools for data visualization have been developed for GE1/1. A "light DQM" framework that allows fast and easy visualization of data taking. Also a full DQM (Data Quality Monitoring) system is under development to interplay with central CMS DQM system.

\section{Perspectives}

The whole GE1/1 system will be installed in CMS during long shutdown 2 (LS2) in 20192020. The first operation of the GE1/1 system will be during run 3 between 2021 and 2023, when the LHC expects to deliver $300 \mathrm{fb}^{-1}$ of integrated luminosity. The instantaneous luminosity during this period will be higher, at least twice its nominal luminosity, in preparation for the HL-LHC.

During the Extended Year End Technical Stop (EYETS) between December 2017 and MarchApril 2018 a slice test for GE1/1 will be installed in CMS endcap. Former tests have been performed at irradiation facilities at CERN, their results can be found in [4]. For the slice test 2 to 4 superchambers will be installed and will take data during 2018. It will serve as a test were the detectors, as well as all the involved systems, will be put in real collisions conditions. It will allow to determine how well the detector performs and what has to be improved before full GE1/1 installation.

\section{Acknowledgments}

We gratefully acknowledge the support of COLCIENCIAS, the administrative department of science, technology and innovation of Colombia.

\section{References}

[1] A. Colaleo, and A. Safonov, and A. Sharma, and M. Tytgat, CMS Technical Design Report for the Muon Endcap GEM Upgrade, CERN-LHCC-2015-012, CMS-TDR-013.

[2] J.A. Merlin, and JM. Brom, and A. Sharma, Study of long-term sustained operation of gaseous detectors for the high rate environment in CMS, CERN-THESIS-2016-041.

[3] V. Brigljevic et al., Using XDAQ in application scenarios of the CMS experiment, in proceedings of eConf C 0303241 (2003) MOGT008 [hep-ex/ 0305076 ].

[4] I. Vai, Test beam and irradiation test results of Triple-GEM detector prototypes for the upgrade of the Muon System of the CMS experiment, CMS-CR-2015-116. 